\title{
Risk Factors for Post-Operative Thyroid Related Complications in Patient Undergoing Thyroidectomy: A Single Center Study Fatemah Saleh AlTheyab ${ }^{1}$, Renad Nasser AlOnazi ${ }^{1}$, Ibtehaj Mohammed AlHarbi ${ }^{1}$, Hessa Mohammed AlHarbi ${ }^{1}$, Sultan AlSaigh ${ }^{2}$ \\ 1.Collage of Medicine, Qassim University, Saudi Arabia, 2. General Surgery Consultant, Head of General Surgery Department King Fahad Specialist Hospital-Buraidah. \\ Corresponding Author: Fatemah Saleh AlTheyab, Tel: 00966 565472636, Email: fatimah.saleh23394@ gmail.com
}

\begin{abstract}
Background: Thyroid diseases are one of the most prevalent endocrinopathies globally ${ }^{(1)}$. Most of the thyroid diseases treated surgically. The operation duration is under multiple factors can be predicted preoperatively ${ }^{(2)}$. Hypocalcaemia and nerve injury are serious complication after thyroid surgery and we hypothesized it could be prevented by simple measures to avoid such complications.
\end{abstract}

Objective: Was to assess the correlation between thyroidectomy operating time, histo-pathological diagnosis of thyroid disease and pre-operative vitamin D supplementation with post thyroidectomy complications mainly hypocalcaemia and nerve injury. Method: An observational retrospective cohort study with a total of 187 patients who underwent thyroidectomy from October $7^{\text {th }} 2013$ to January $4^{\text {th }} 2018$ were included in this study. Data were analyzed for demographic information including age, sex, nationality, height and weight. Information about the use of vitamin D supplementation before or after the operation, the clinical and the histological diagnosis of thyroid diseases, the name of the procedure (partial or total thyroidectomy), and the presence of any complications. Result: The mean age of the patients was 39.7 \pm 12.71 years. $81.8 \%(153)$ were females and $18.2 \%(34)$ were males. 53.5\%(100) were given Vitamin-D supplements before or after the surgery. Histopathology showed that the most prevalent type was papillary Carcinoma accounting for 68 (36.4\%). No significant association between histological diagnosis and complication of hypocalcaemia and nerve injury. Mean duration of surgery performed was $135.61 \pm 47.668$. 83 of our patients suffered from hypocalcaemia and 6 suffered from nerve injury. When correlating between duration of surgery with hypocalcaemia and nerve injury, The independent t-test was associated with statistically insignificant effect $\mathrm{t}(182)=-1.85, \mathrm{p}=0.066$ $(>0.05)$ for hypocalcaemia and $t(185)=-0.075, p=0.940(>0.05)$ for nerve injury.

Conclusion: Our study suggested that there was no significant correlation between the duration of surgery as well as histo-pathological diagnosis and the development of post-operative hypocalcaemia and nerve injury.

Keywords: Thyroidectomy, Post-operative hypocalcaemia, Post-operative nerve injury, Complications, Vitamin D.

\section{INTRODUCTION}

Thyroid diseases are one of the most prevalent endocrinopathies globally, however, the importance of thyroid diseases is recently identified and now is considered as one of the non-communicable diseases that affect the community. In Saudi Arabia thyroid ranked as fourth in male and second in female as most prevalent cancer and it represents $6.1 \%$ of newly diagnosed cancers, thyroid cancer is the most common endocrine malignancy and it accounts $87 \%$ of all endocrine gland cancers ${ }^{(1)}$. Most of the thyroid diseases treated surgically especially when medical treatment fails, cosmetic purposes or when thyroid cancer extensively invading the surrounding structures. The operation duration is under multiple factors can be predicted preoperatively ${ }^{(2)}$. A study was conducted in tertiary referral center state that operation duration more than $120 \mathrm{~min}$ will lead to hypocalcaemia ${ }^{(3)}$. Where was another study stated that there was no correlation between long operation duration and postoperative complications (4). Another factor for postoperative hypocalcaemia is vitamin D preoperative and postoperative supplements. A randomized controlled trial of alfacalcidol supplementation. The results showed that preoperative and postoperative supplementation decreased transient hypocalcaemia (5). Another randomized controlled trial was conducted to assess the efficiency of preoperative and postoperative calcium and vitamin D supplementation to prevent postoperative hypocalcaemia. The results confirmed decreased postoperative hypocalcaemia ${ }^{(6)}$. A systematic review and meta-analysis reported a significant decrease in postoperative hypocalcaemia in patients who received oral calcium or vitamin D supplementation ${ }^{(7)}$. Hypocalcaemia and nerve injury were serious complication after thyroid surgery and we hypothesized it could be prevented by simple measures to avoid such complications. Our aim of this study was to estimate the relationship between thyroidectomy operating time and postoperative complications mainly hypocalcaemia and nerve injury and assess the correlation between 
preoperative vitamin D supplementation and postthyroidectomy hypocalcaemia.

\section{METHODOLOGY \\ Procedures}

The present research was an observational retrospective cohort study to measure the correlation between operating time and post thyroidectomy complications - mainly hypocalcemia and nerve injury-. A chart review of 187 files of patients undergoing thyroidectomy in King Fahad Specialist Hospital (KFSH) was done. KFSH was the only tertiary care hospital in Qassim region with referrals from all governmental hospitals in the region.

\section{Measurement}

The sheet used for collecting data included demographic information including age, sex, and nationality. We also included the file number to avoid duplication of data. Height and weight were also collected from patients' files. Information about the use of vitamin D supplementation before or after the operation, the clinical and the histological diagnosis of thyroid diseases, the name of the procedure (partial or total thyroidectomy), and the presence of any complications were included in the datasheet. All this information was based on previous medical literature ${ }^{(1,4)}$ and the sheet was validated by a senior, the head of surgical department in KFSH.

\section{Data Collection}

Collection of data was started in January 2018, after the IRB approval was obtained from Qassim ethical committee and after hospital approval to conduct this study. Data were gathered from the General Surgery department of KFSH and we included all files from October $7^{\text {th }} 2013$ to January $4^{\text {th }} 2018$. Files with missing identification data or patients undergoing procedures other than thyroid surgery were excluded. Data were collected on Excel files and later on, were exported to SPSS program version 21 for analysis.

\section{Statistical Analysis}

Both descriptive and inferential statistics were done to determine the association between variables. Descriptive statistics included frequencies, counts, and percentages were presented in tables. Regarding inferential statistics, an independent t-test was performed to study the association between surgery duration and the development of postoperative complications.

\section{RESULTS}

Data from total of 187 patients. 7\% (13) nonSaudi nationals and 93\% (174) Saudi nationals was selected to be included in the study from hospital records of thyroid surgeries. The mean age of the patients was $39.7 \pm 12.71$ years, mean height was $157.65 \pm 8.407 \mathrm{~cm}$. and mean weight was $74.06 \pm 18.731 \mathrm{~kg}$. Out of total $81.8 \%$ (153) patients were females and $18.2 \%$ (34) were males. It was calculated that for most of the patient's total thyroidectomy was chosen as the procedure $88.24 \%$ (165). Out of total 56 patients who developed postoperative complications, complications of 52 patients were resolved at the time of discharge from the hospital. Mean post-operative stay in hospital was 4.04 \pm 3.764 days. 53.5\%(100) were given Vitamin-D supplements before or after the surgery and $46.5 \%(87)$ were not given any vitamin D supplement. While addressing complication of hypocalcaemia 34 patients were given calcium carbonate and 20 were given calcium gluconate either alone, combined or with other treatment options.

After performing the histopathology, papillary Carcinoma 68 (36.4\%) was found most common in our patients. When correlation between histological diagnosis and complication of hypocalcaemia and nerve injury were studied, the results don't show any significant relationship between histopathology and complication (Table1). More study is needed in this aspect of thyroid histopathology. Mean duration of surgery performed $135.61 \pm 47.668$ and most of the surgeries i.e. $111(59.4 \%)$ were performed within time limit of 90-150 minutes (Table 2). During surgery only 2 patients had a complication of blood loss no other complication were documented. Among 187(N) patients 83 suffered from hypocalcaemia and 6 suffered from nerve injury. By comparison, there was no association between duration of surgery and complications. To test the hypothesis that the increase in the duration of surgery (135.61 \pm 47.668$)$ and complications are not associated statistically, an independent sample t-test was performed. As it can be seen in (Table 3) that duration of surgery is distributed sufficiently normal for the purpose of conducting a t-test (i.e., skew $=1.255$ and kurtosis 2.693) .

For association between duration of surgery with hypocalcaemia and nerve injury, the assumption of homogeneity of variance was tested and satisfied via Levene's F test, $\mathrm{F}(182)=2.661, \mathrm{p}=0.105(>0.05)$ and $\mathrm{F}(185)=1.841, \mathrm{p}=0.176(>0.05)$ respectively. The independent t-test was associated with statistically insignificant effect $\mathrm{t}(182)=-1.85, \mathrm{p}=0.066(>0.05)$ for hypocalcaemia and $\mathrm{t}(185)=-0.075, \mathrm{p}=0.940$ $(>0.05)$ for nerve injury (Table 4,5) . Thus, the duration of surgery has no statistical association to the hypocalcaemia and nerve injury (Figure 1,2). The null hypothesis stays valid and showed that there was no relation between duration of surgery and complications. 
Table 1. Correlation between histo-pathological type and post-operative complications.

\begin{tabular}{|c|c|c|c|c|c|c|c|}
\hline \multicolumn{8}{|c|}{ Relationship between Histopathology and Complications } \\
\hline & & \multirow{2}{*}{$\mathbf{N}$} & \multirow{2}{*}{$\begin{array}{l}\text { Percent } \\
\%\end{array}$} & \multicolumn{2}{|c|}{ hypocalcaemia } & \multicolumn{2}{|c|}{ Nerve injury } \\
\hline & & & & Developed & $\%$ & Developed & $\%$ \\
\hline \multirow{10}{*}{$\begin{array}{l}\text { Histological } \\
\text { Diagnosis }\end{array}$} & Papillary Carcinoma & 68 & 36.36 & 27 & $39.71 \%$ & 2 & $3 \%$ \\
\hline & Follicular Carcinoma & 9 & 4.81 & 6 & $66.67 \%$ & 0 & $0 \%$ \\
\hline & Follicular Adenoma & 10 & 5.35 & 2 & $20.00 \%$ & 1 & $10 \%$ \\
\hline & Benign & 21 & 11.23 & 8 & $38.10 \%$ & 1 & $5 \%$ \\
\hline & Hurthle Cell Adenoma & 3 & 1.60 & 1 & $33.33 \%$ & 0 & $0 \%$ \\
\hline & Hurthle Cell Carcinoma & 5 & 2.67 & 1 & $20.00 \%$ & 0 & $0 \%$ \\
\hline & Non-Hodgkin Lymphoma & 1 & 0.53 & 1 & $100.00 \%$ & 0 & $0 \%$ \\
\hline & Simple Goiter & 40 & 21.39 & 22 & $55.00 \%$ & 1 & $3 \%$ \\
\hline & Toxic Goiter & 1 & 0.53 & 1 & $100.00 \%$ & 0 & $0 \%$ \\
\hline & Hashimoto Thyroiditis & 23 & 12.3 & 11 & $47.83 \%$ & 1 & $4 \%$ \\
\hline
\end{tabular}

Table 2. Duration of thyroidectomy

\begin{tabular}{|l|l|}
\hline Duration of surgery (Minutes) & Frequency \\
\hline $31-60$ & 3 \\
\hline $61-90$ & 24 \\
\hline $91-120$ & 57 \\
\hline $121-150$ & 54 \\
\hline $151-180$ & 28 \\
\hline $181-210$ & 8 \\
\hline $211-240$ & 8 \\
\hline $241-270$ & 2 \\
\hline $271-300$ & 1 \\
\hline $301-330$ & 1 \\
\hline $331+$ & 1 \\
\hline Grand Total & $\mathbf{1 8 7}$ \\
\hline
\end{tabular}

Table 3. Correlation between duration of surgery and post-operative complication

\begin{tabular}{|l|l|l|l|l|l|l|}
\hline \multirow{2}{*}{ Descriptive Statistics Associated with Duration of surgery and complications } \\
\cline { 2 - 7 } & N & Mean & \multicolumn{2}{|c|}{ Skewness } & \multicolumn{2}{l|}{ Kurtosis } \\
\hline Statistic & Statistic & Statistic & Std. Erro & Statistic & Std. Error \\
\hline Duration Of Surgery(min) & 187 & 135.61 & 1.255 & .178 & 2.693 & .354 \\
\hline hypocalcaemia & 187 & .57 & .040 & .178 & -1.276 & .354 \\
\hline Nerve Injury & 187 & .97 & -5.353 & .178 & 26.947 & .354 \\
\hline
\end{tabular}

Table 4. Correlation between duration of surgery and hypocalcaemia

T-test (Duration of Surgery and Hypocalcaemia)

\begin{tabular}{|l|l|l|l|l|l|}
\hline & Hypocalcaemia & N & Mean & Std. Deviation & Sig. (2-tailed) \\
\hline \multirow{2}{*}{ Duration of surgery(min) } & No & 101 & 130.10 & 22.210 & 182 \\
\cline { 2 - 6 } & Yes & 83 & 143.13 & 23.322 & 154.584 \\
\hline
\end{tabular}

Table 5. Correlation between duration of surgery and nerve injury

\begin{tabular}{|l|l|l|l|l|l|}
\hline T-test (Duration of Surgery and Nerve Injury) \\
\hline & Nerve Injury & N & Mean & Std. Deviation & Sig. (2-tailed) \\
\hline \multirow{2}{*}{ Duration of Surgery(min) } & Yes & 6 & 134.17 & 20.837 & 0.940 \\
\cline { 2 - 6 } & No & 181 & 135.66 & 48.330 & 0.876 \\
\hline
\end{tabular}


Risk Factors for Post-Operative Thyroid Related Complications...

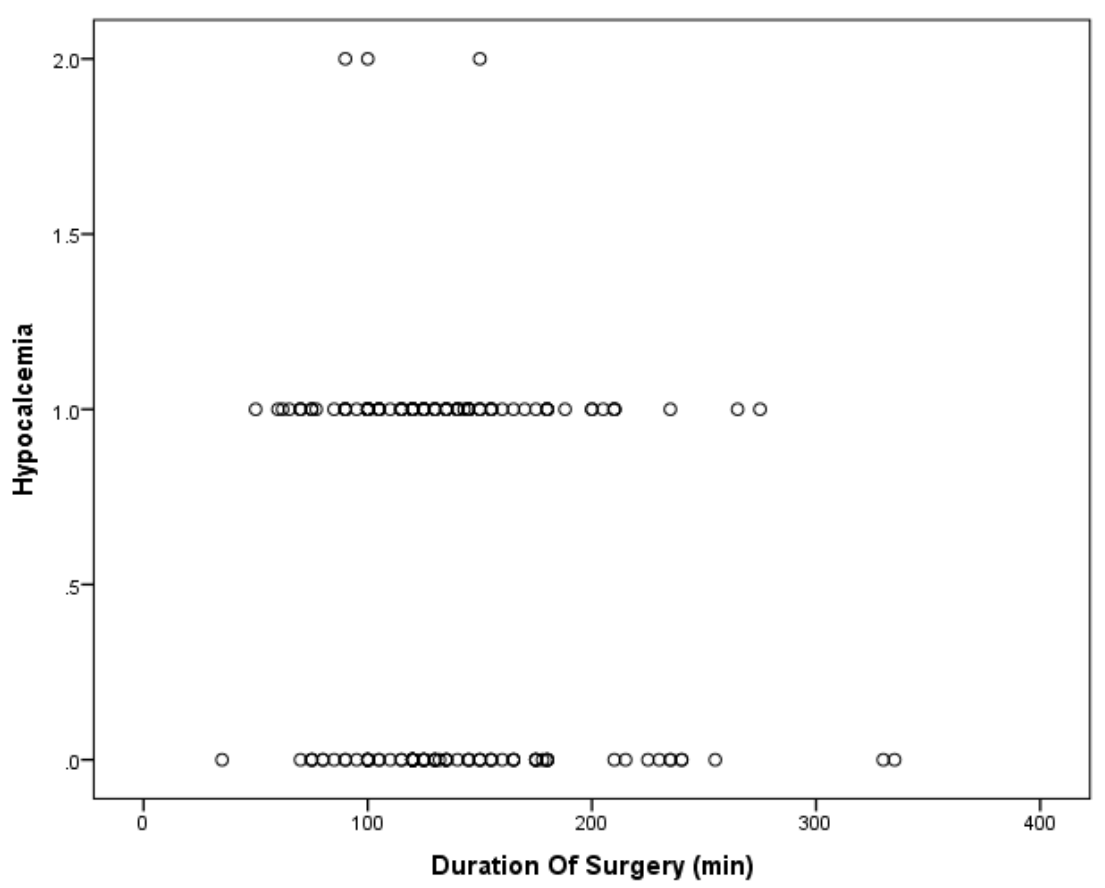

Figure 1. Association between the duration of surgery and complication of hypoglycemia $\left(\mathrm{p}=0.044,-.148^{*}\right)$.

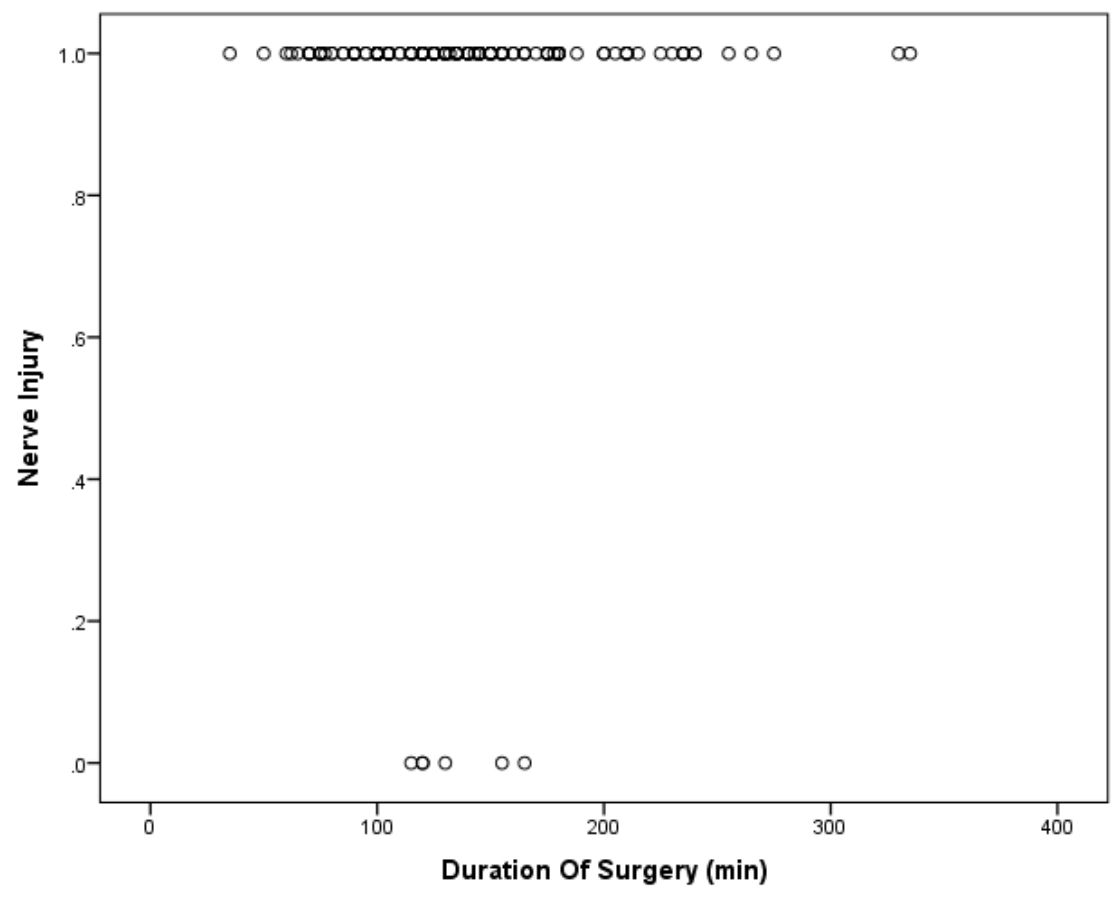

Figure 2. Association between the duration of surgery and nerve injury ( $\left.\mathrm{p}=0.940,0.006^{*}\right)$. 


\section{DISCUSSION}

Hypocalcaemia and nerve injury are the most concerning complications following thyroidectomy. In this study, we aimed to see the effect of thyroidectomy operating time and the development of postoperative complications mainly hypocalcaemia and nerve injury. Our study showed that most of the patients were females $(81.8 \%)$, and the mean age for the patients was 39.7 years which was consistent with the findings of a study that was previously done in the eastern province in Saudi Arabia ${ }^{(1)}$.

Papillary thyroid carcinoma has been reported as the most common type among other histological pattern ${ }^{(1)}$. The histopathology results in our study as well showed that papillary thyroid carcinoma was the most prevalent type accounting for $36.4 \%$ of the sample followed by simple goiter and Hashimoto thyroiditis. Considering the histological type as a risk factor for developing post-operative hypocalcaemia and nerve injury, the analytical study showed no significant correlation. Rather than the histological type, Peter C Ambe had suggested that recurrent retrosternal and intrathoracic goiter may contribute to the development of post-operative hypocalcaemia and nerve injury (4). In case of recurrent goiter, Hazem M. Zakaria justified that adhesions and anatomical displacement are the main reasons for developing post-operative nerve injury ${ }^{(9)}$. Also, in his study which was specifically discussing recurrent laryngeal nerve injury. He reported that the histopathological diagnosis is a factor that can lead to nerve injury in addition to other factors ${ }^{(9)}$.

According to American Thyroid Association, the duration for thyroidectomy range from 45 minutes to 3 hours which agrees with our findings with a mean duration of $135.61 \pm 47.668$ minutes ${ }^{(10)}$. The variety in the operation length can be explained mainly by the surgeon's experience instead of what previously thought that the patient factors play an important role as Patoir had suggested (2). When correlating the operating time and post-operative complications mainly hypocalcaemia and nerve injury, there is no significant positive relationship in between, which has been reported previously that the increase in length of the surgery is not a risk factor for complications development ${ }^{(4)}$. In contrast, a study done by Emilie Sonne-Holm demonstrated that longer surgery time $(>120$ minutes ) is an influential factor for post-operative hyperparathyroidism due to ischemia of the parathyroid gland which will lead subsequently to hypocalcaemia ${ }^{(3)}$.

Previous studies reported that vitamin D in addition to calcium supplementation is a protective factor from post-operative development of hypocalcaemia ${ }^{(11)}$. However, supplements are not usually given to all patients due to the possibility of adverse effects ${ }^{(11)}$. A randomized control study suggested that pre-operative vitamin D supplementation 1 day prior to surgery and 8 days following the surgery significantly have reduced the incidence of post-operative hypocalcaemia as well as accelerate the baseline return of calcium level ${ }^{(5)}$.

Another study suggested the same result but supplementation of vitamin D was provided 7 days before and 7 days after the surgery ${ }^{(6)}$. Amal Alhefdh as well demonstrated that further supplementation of vitamin $\mathrm{D}$ in addition to calcium significantly reduced the incidence of hypocalcaemia, but she suggested that further studies are needed to determine the dose and if it should be given pre or post operatively ${ }^{(7)}$. Our concern is to determine if pre-operative vitamin D supplementation can reduce the incidence of postoperative hypocalcaemia. But we have major limitations because only $53.5 \%$ of our patients were given vitamin $\mathrm{D}$ and most of them were given the supplementation post-operatively along with calcium. For those who were given pre-operatively, the duration as well as the dose was different among the patients, also the form of vitamin D that was supplemented differ from one patient to another. For these reasons it was difficult to compare the effectiveness of the supplementation. Due to the retrospective nature of the study, there was troublesome to determine the reason for vitamin D supplementation pre-operatively so other co-morbidities that can affect the result cannot be excluded. We suggest a prospective study to be conducted comparing two groups of patients; one with pre-operative and the other with post-operative supplementation putting in mind that both groups will receive the same form, dose and duration of supplementation with exclusion of other comorbidities.

In addition to the retrospective nature of the study, our limitations include: a relatively small sample size, and lack of detailed information of vitamin D supplementation which need to obtained such as the dose, duration and presence of other comorbidities that need vitamin D as treatment. Also, our study was conducted in a single center and the results cannot be generalized among all centers.

\section{Conclusion}

Our study suggests that there was no significant correlation between the duration of surgery as well as histo-pathological diagnosis and the development of post-operative hypocalcaemia 
and nerve injury.

\section{ACKNOWLEDGMENT}

I would like to acknowledge Dr. Jaber AlHarbi for helping in the conceptualization of the research idea and Dr. Arshad Malik for his advice throughout the research.

\section{REFERENCES}

1. Al-Amri AM(2012): Pattern of Thyroid Cancer in the Eastern Province of Saudi Arabia: University Hospital Experience. Journal of Cancer Therapy, 3(03):187-191.

2. Patoir A, Payet C, Peix J et al.(2017): Determinants of operative time in thyroid surgery: A prospective multicenter study of 3454 thyroidectomies. Plos One, doi:10.1371/journal.pone.0181424.

3. Sonne-Holm E, Holst H (2017): Prolonged Duration of Surgery Predicts Postoperative Hypoparathyroidism among Patients Undergoing Total Thyroidectomy in a Tertiary Referral Centre. European Thyroid Journal, 6(5):255-262.

4. Ambe PC, Brömling S, Knoefel WT, Rehders A(2014): Prolonged duration of surgery is not a risk factor for postoperative complications in patients undergoing total thyroidectomy: a single center experience in 305 patients. Patient Safety in Surgery. $8(1): 45$.
5. Genser L, Trésallet $\mathrm{C}$, Godiris-Petit $\mathrm{G}$ et al.(2014): Randomized controlled trial of alfacalcidol supplementation for the reduction of hypocalcemia after total thyroidectomy. The American Journal of Surgery, 207(1):39-45.

6. Laway B, Jaan S, Sehgal A, Wani R, Wani M, Wani $\mathbf{K}(2017)$ : Usefulness of pre- and post-operative calcium and Vitamin D supplementation in prevention of hypocalcemia after total thyroidectomy: A randomized controlled trial. Indian Journal of Endocrinology and Metabolism, 21(1):51.

7. Alhefdhi A, Mazeh H, Chen H(2013): Role of Postoperative Vitamin D and/or Calcium Routine Supplementation in Preventing Hypocalcemia After Thyroidectomy: A Systematic Review and MetaAnalysis. The Oncologist,18(5):533-542.

8. Schmider E, Ziegler M, Danay E, Beyer L, Bühner M(2010): Is It Really Robust? Methodology, 6(4):147151.

9. Zakaria A(2011): Recurrent Laryngeal Nerve Injury in Thyroid Surgery. Oman Medical Journal, 26(1):34-38.

10. Q and A: Thyroidectomy. American Thyroid Association(2018) https://www.thyroid.org/patientthyroid-information/what-are-thyroid-problems/q-anda-thyroidectomy/. Accessed March 23.

11. Noureldine SI, Genther DJ, Lopez M, Agrawal N, Tufano RP(2014): Early Predictors of Hypocalcemia After Total Thyroidectomy. JAMA OtolaryngologyHead \& Neck Surgery, 140(11):1006. 